(C) 2016 IEEE. Personal use of this material is permitted. Permission from IEEE must be obtained for all other uses, in any current or future media, including reprinting/republishing this material for advertising or promotional purposes, creating new collective works, for resale or redistribution to servers or lists, or reuse of any copyrighted component of this work in other works. 


\title{
Performance of well-known frequency reuse algorithms in LTE downlink 3GPP LTE systems
}

\author{
Sinh Cong Lam, Ramprasad Subramanian, Kumbesan Sandrasegaran, Pantha Ghosal, Shouman Barua \\ Centre for Real-Time Information Networks, Faculty of Engineering and Information Technology, \\ University of Technology, Sydney, Australia
}

\begin{abstract}
Intercell interference (ICI) is one of the major factors that limit the performance of wireless cellular network systems. Soft frequency reuse (SFR) as well as its modified algorithms such as Soft fractional frequency reuse (Soft FFR) and Distributed fractional frequency reuse (Distributed FFR) have been introduced as an effective way to optimize spectrum and control the ICI. However, the comparison between these algorithms has not fully been presented by the researchers proposing the models. This paper presents a comparison of the performance of well-known frequency reuse algorithms in term of system throughput, average packet loss ratio and average packet delay. The simulation results indicate that the simplest scheme, i.e. Soft FR, archives the highest system performance comparing to Soft FFR and Distributed FFR. Hence, it is noticed that one of the effective methods which optimize frequency reuse is to reduce the algorithm complexity.
\end{abstract}

Key words: Distributed frequency reuse, Soft frequency reuse, Soft fractional frequency reuse, network simulation v3 NS3.

\section{INTRODUCTION}

In Orthogonal Frequency Division Multiple (OFDM) multi-cell networks, the main factor, directly impacting on the system performance, is intercell interference which causes by the use of the same frequency band in adjacent cells. Intercell interference coordination (ICIC)[1] has been promised as a technique that can significantly mitigate the intercell interference then improve network performance, especially for users suffering low Signal-toInterference-Noise ratio (SINR). Generally, ICI techniques can be partitioned into two schemes, called interference mitigation and avoidance.

Interference mitigation aims to reduce or suppress the intercell interference during transmission or at the receivers. The interference mitigation techniques include interference averaging, interference cancelation and adaptive beamforming [2, 3]. Interference averaging was introduced in an effort to randomize the interfering signals among all users by scrambling the codeword at the transmitters and the processing again at the receivers. This technique is quite simple and does not require additional measurements and signaling. However, this technique does not meet EUTRA requirements because it does not improve the signal strength of cell-edge users (CEUs). ICI cancellation attempts to suppress the interference by using the processing gain at the receivers. This technique estimates the interfering signal based on the previous receptions and then subtracts the estimated interfering signal from the received signal to receive more reliable signal. ICI cancellation does not improve the strength of desired signal, but it can improve the quality of signal. Hence, this technique can improve the performance. However, it requires the knowledge of interference statistics and additional complexity at the receivers. Adaptive beamforming utilizes smart antennas which can automatically change their radiation patterns to improve the signal strength. This technique is quite complex and requires additional hardware elements.

Interference Avoidance refers frequency reuse algorithms which are used to deploy some restrictions on the transmission power and resource allocation. The aims of ICI avoidance are to reduce the intercell interference as well as to improve the desired signal strength. Specially, this scheme does not impose any additional computation as well as extra hardware element on the user's device. Hence, it is promised as the most effective technique for LTE network to provide high quality of services to cell-edge user without sacrificing cell-center performance.

In most previous works on Interference avoidance, the authors proposed the new frequency reuse algorithms to optimize a specific parameter such as transmission power, intercell interference [4] or cell throughput[1, 5]. However, other parameters of cellular network performance such as average packet loss and average packet delay were not compared together. Due to this omission, it might be unfair to compare the frequency reuse algorithms because the more complex algorithms need more additional processing latency. This means if a delay threshold is assigned for each packet, the complex algorithms can produce higher packet loss ratio than the simple ones. To give an overall comparison, this paper compares the three wellknown frequency reuse algorithms, called Soft FR, Soft FFR, and Distributed FFR by both mathematical logic and simulation.

\section{FREQUENCY REUSE ALGORITHMS}

In this session, Strict FR, Soft FR, Soft FFR, and Distributed FFR are discussed and compared in the context of a system with $\mathrm{M}+1$ hexagonal cells and 
every cell is allocated the same $\mathrm{N}$ sub-bands. It is assumed that frequency reuse factor $\Delta$ is deployed and $\mathrm{N}$ available sub-bands are partitioned into two groups having $\mathrm{N}_{\mathrm{C}}$ and $\mathrm{N}_{\mathrm{E}}$ sub-bands $\left(\mathrm{N}_{\mathrm{C}}+\mathrm{N}_{\mathrm{E}}=\mathrm{N}\right)$. We define that the maximum numbers of interfering base stations is the set of base stations that can cause intercell interference on a typical user. The interfering probability is defined as the probability in which a base station cause interference on a typical user.

\subsection{Strict frequency reuse}

In strict FR with reuse factor $\Delta=3$ as in Figure 1, the whole bandwidth is dived into one common sub-band and $\Delta$ private sub-band group. The common sub-bands are allocated to cell-center area with low power level in every cell while each private sub-band group is allocated to the cell-edge area at high power level. In this case, the cell-center users (CCUs) are allowed to use $\mathrm{N}_{\mathrm{C}}$ sub-bands, while the cell-edge users in each cell are only allowed to use $\frac{\mathrm{N}_{\mathrm{E}}}{\Delta}$. Hence, the interfering probabilities for a cell-edge and cellcenter user are $\frac{1}{\mathrm{~N}_{\mathrm{c}}}$ and $\frac{\Delta}{N_{E}}$, respectively.

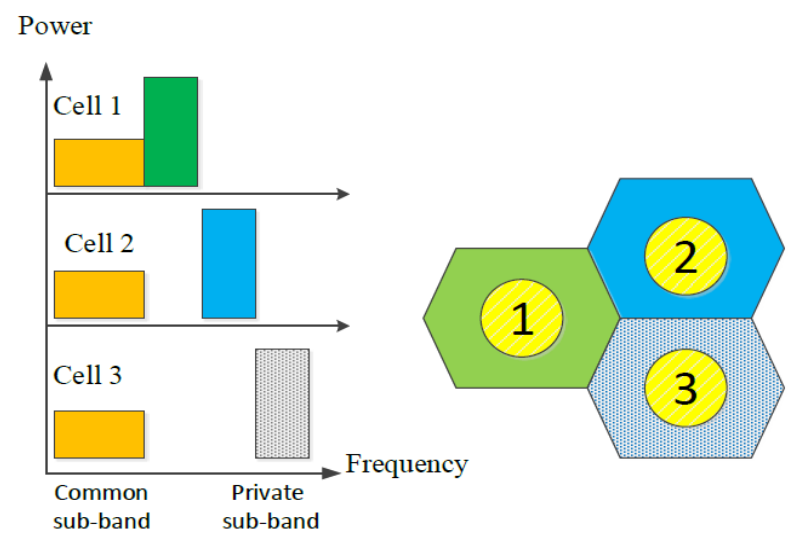

Figure 1: Strict frequency reuse

It is noticed that cell-center users do not share their own sub-bands to the cell-edge users, and then a cell-center (cell-edge) user is only affected by interfering that is cause by base station transmitting on the same cell-center (cell-edge) sub-band. Hence, there are $\mathrm{M}$ interfering cells that can effect on interior users, but there are only $\frac{\mathrm{M}}{\Delta}$ for the case of exterior users. The interferences that cause both cell-edge and cell-center users is, hence, minimized.

\subsection{Soft frequency reuse}

Soft FR scheme (SFR) [6] is a modification of Strict FR in which the whole bandwidth is divided into two sub-bands, called the cell-edge and cell-center subbands. The main difference between Strict and Soft FR is that the cell-center users share the allocated subbands with cell-edge users in adjacent cells in an effort to improve spectrum efficiency and system performance.

\section{Power}

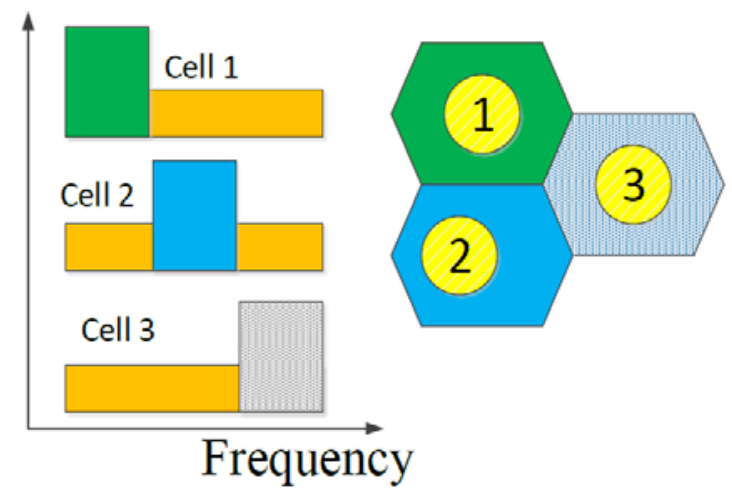

Figure 2: Soft frequency reuse with reuse factor $\Delta=3$

Because of sharing resource between cell-edge and cell-center users, cell-edge users can use the whole cell-edge sub-bands. Hence, the interfering probabilities for a cell-center and cell-edge user are, respectively, $\frac{1}{\mathrm{~N}_{\mathrm{C}}}$ and $\frac{1}{\mathrm{~N}_{\mathrm{E}}}$. The maximum interfering cells for both cases of users are the same and equal $M$ in which $\frac{(\Delta-1)}{\Delta} M$ base stations transmitting on cell-center sub-bands at low power level and $\frac{1}{\Delta} \mathrm{M}$ base stations transmitting on celledge sub-bands at high power level. Therefore, it is said that Soft FR can produce more interference on a user than Strict FR.

\subsection{Soft fractional frequency reuse}

Soft fractional frequency reuse scheme (SFFR) was introduced in [5] as a combination of Strict and Soft to increase the cell-center user throughput without increasing the power consumption [7]. In this scheme, the allocated edge bandwidth is divided into two nonoverlapping sub-bands called $N_{C}^{\prime}$ center, $N_{M}$ medium and $N_{E}^{\prime}$ edge sub-bands $\left(N_{C}^{\prime}+N_{M}+N_{E}^{\prime}=N\right)$. The medium sub-bands (yellow color) work as cell-center sub-bands in Strict FR and two others such as medium and edge sub-bands work as cell-center and cell-edge sub-bands in Soft FR [8]. The maximum interferes for a typical user in each cell area is similar in Strict and Soft FR.
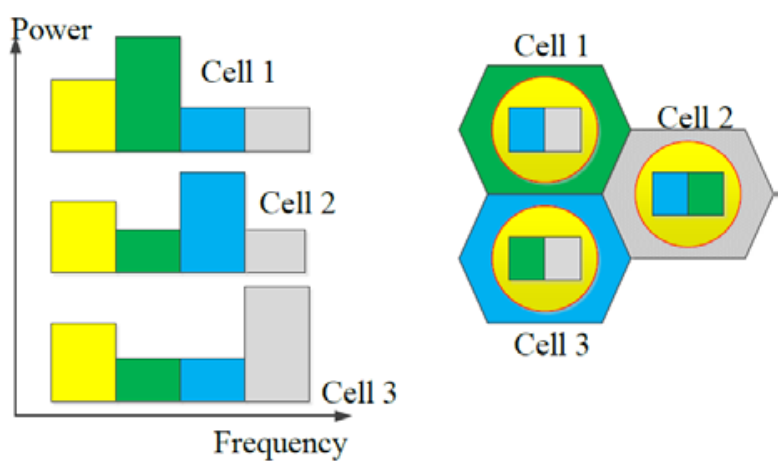

Figure 3: Soft fractional frequency reuse with factor

$$
\Delta=3
$$


In the Soft FFR and Soft FR algorithms, the usage of resource blocks in the adjacent cells is not considered. This leads to an increase in intercell interference which occurs when one resource block (RB) is used by two neighboring cells at the same time.

\subsection{Distributed fractional frequency reuse}

Distributed fractional frequency reuse (Distributed FFR) algorithm [4] was proposed to minimize the effect of ICI by exchanging ICI information between the adjacent cells through $\mathrm{X} 2$ interface. The eNodeB estimates the quality of channel $j$ based on the cell-edge-band matrix $C_{i j}$ [4]

$$
C_{i j}=\sum_{i \in I} \delta_{i j} w_{i}
$$

In which: $\delta_{i j}$ takes a value of 1 if $\mathrm{RB} j$ is occupied at cell edge and 0 otherwise and $w_{i}$ denotes the estimated number of UEs at adjacent cell $i$. If the difference between the power of interfering signal and desired signal is larger than the threshold, $w_{i}$ will be increased by one.

Sub-channel for which matrix $C_{i j}$ is the largest is considered to be strongest effected by ICI then assigned the lowest priority for transmitting. Hence, Distributed FFR can reduce the probability in which two adjacent cells transmit on the same resource block at the same time. As a result, the interfering probabilities and the maximum number of interfering base stations are smaller than in Soft FR.

The differences between these algorithms can be summarized in Table 1. It is clear that Distributed FR can provide the highest SINR to associated users and then it can provide the highest system throughput. However, it is reminded that this throughput is analyzed without considering the impact of transmission and processing delay. The overall performance of these algorithms will be discussed in session 3 and 4 .

\section{SIMULATION MODEL}

The network model is shown in Figure 4 in which users exchange information with the remote host [9] through radio channels, point to point links and internet connections. The wireless channels between users and eNodeBs are modeled as in [10] in which the transmitted signals have to experience propagation path loss, fast fading, slow fading as well as Doppler effect. The connections from eNodeB to remote host are assumed to have unlimited bandwidth and then the transmission delay and packets loss on these links can be neglected. Hence, this network model can be used to evaluate the performance of radio links between users and eNodeBs.

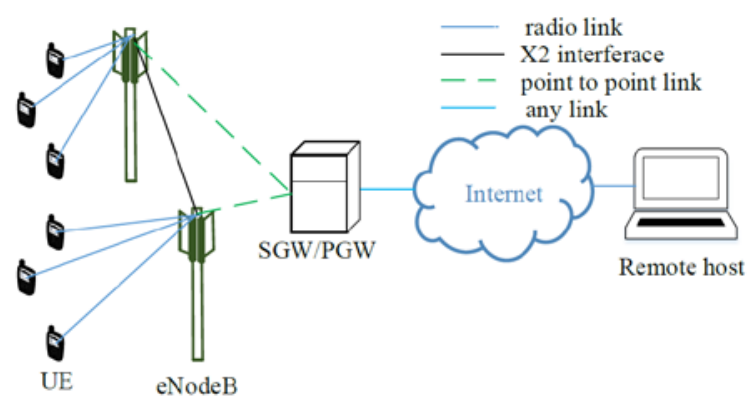

Figure 4: Network model [9]

To examine the well-known frequency reuse algorithms, a 19 hexagonal cell model with frequency reuse factor of 3 at the cell-edge and 1 at the cell-center is considered. UEs are normally distributed within each cell moving randomly at a speed of $3 \mathrm{~km} / \mathrm{h}$ and have internet connections to exchange 72 byte packets (e.g. VoIP) with the remote host $[9,11]$. The simulation parameters are summarized in Table 2

\begin{tabular}{|l|l|}
\hline Parameters & Value \\
\hline Carrier frequency & $2 \mathrm{GHz}$ \\
\hline Bandwidth & $5 \mathrm{MHz}$ \\
\hline Number of RBs & 25 \\
\hline Number of cells & 19 \\
\hline Cell radius & $1000 \mathrm{~m}$ \\
\hline Packet interval & $20 \mathrm{~ms}$ \\
\hline Packet size & 72 bytes \\
\hline Fading model & Extended Pedestrian A [10] \\
\hline Scheduling & Proportional Fair \\
\hline UE distribution & Normal distribution, \\
& Moving at 3km/h \\
\hline SINR threshold & $\begin{array}{l}20 \mathrm{~dB} \text { for SFR and distributed FR } \\
25 \mathrm{~dB} \text { and } 15 \mathrm{~dB} \text { for Soft FFR }\end{array}$ \\
\hline Simulation time & 30s \\
\hline
\end{tabular}

Table 2: Simulation parameters

We assume that SFR, distributed FFR and the two outer areas of SFFR use the same power profiles as 3GPP recommendation [12] . The center region of cells enabled Soft FFR is assumed to work with lower power to save energy consumption.

\section{SIMULATION RESULTS}

The system performance parameters including average values of packet delay, packet loss ratio/flow (PLR) and throughput/UE are calculated for the whole system (i.e. 19 cells) and for the worst cell, i.e. center cell which is located at the center of 18 cells. From figures, it is observed that the average performance over the whole system is better than over the worst cell which experiences the higher ICI level. Furthermore, Soft FFR achieves the lowest system performance due to decrease in the transmission power at the center area in Soft FFR which may be not strong enough to combat the fading and inter-cell interference. It is also noticed that in Soft FFR, the entire bandwidth is separated into three small sub-bands and allocated to specific areas which may reduce the flexible of system resource, e.g. 
users are mainly distributed at a center or edge area. On the other hand, Soft FR as well as Distributed FFR allow more RBs to be used at the cell-edge area, and then these schemes can obtain the higher the performance than Soft FFR.

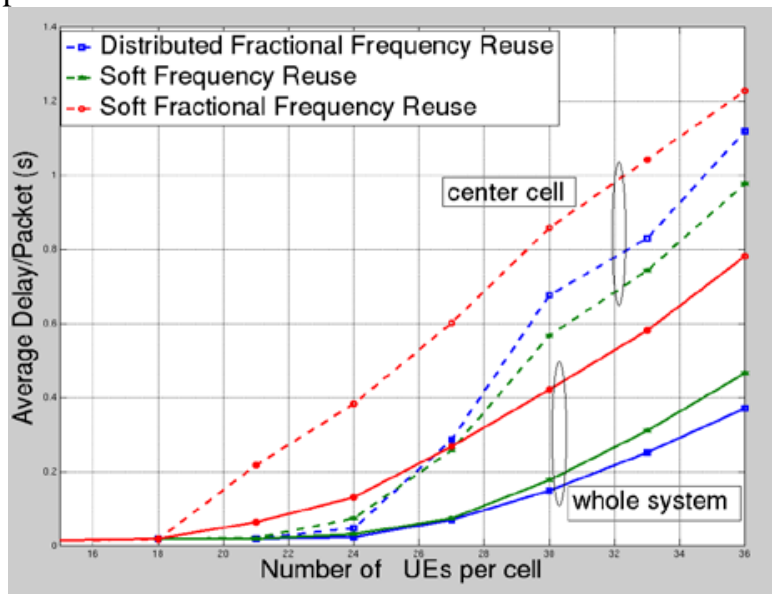

Figure 5: Average Delay/Packet

When compared with SFR, distributed FFR algorithm can reduce the ICI by exchange cell-edgeband matrix $C_{j}$ between each cell with its neighboring cells. This, however, leads to more complex computation required to configure this matrix and broadcast it to all eNodeBs. The simulation indicates that Distributed FFR only outperforms Soft FR in terms of whole system performance with high number of users; otherwise it can be observed that the complex computation causes the higher delay, packet loss ratio and lower throughput than Soft FR.

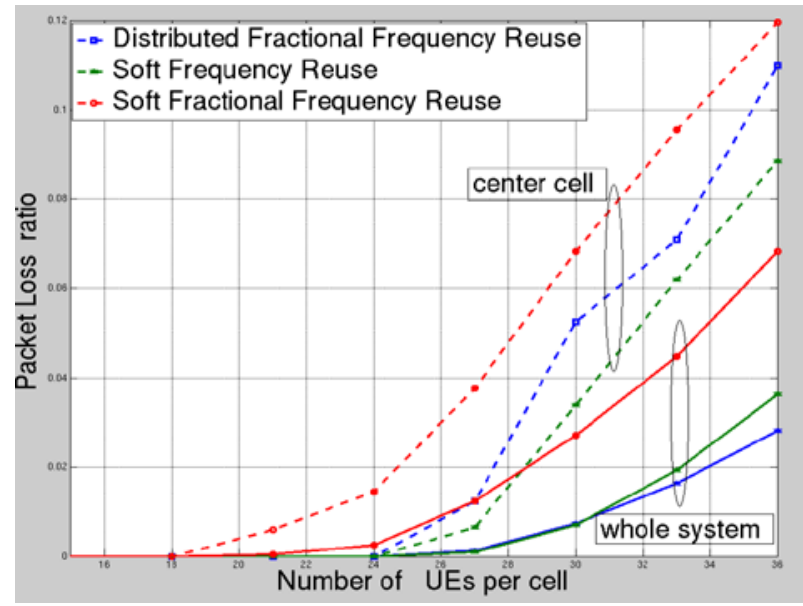

Figure 6: Packet loss ratio per flow

In a VoIP call service environment, it is important to maintain the average delay/packet and packet loss ratio below $100 \mathrm{~ms}$ and $10^{-2}$ [13] such that the QoS requirements of VoIP call service could be met. In Figure 6-7, it can be observed that less than 26 users can be supported by Soft FR and Distributed FFR at the target PLR threshold. On the other hand, Soft FFR can only support around four less number of users at the same QoS.

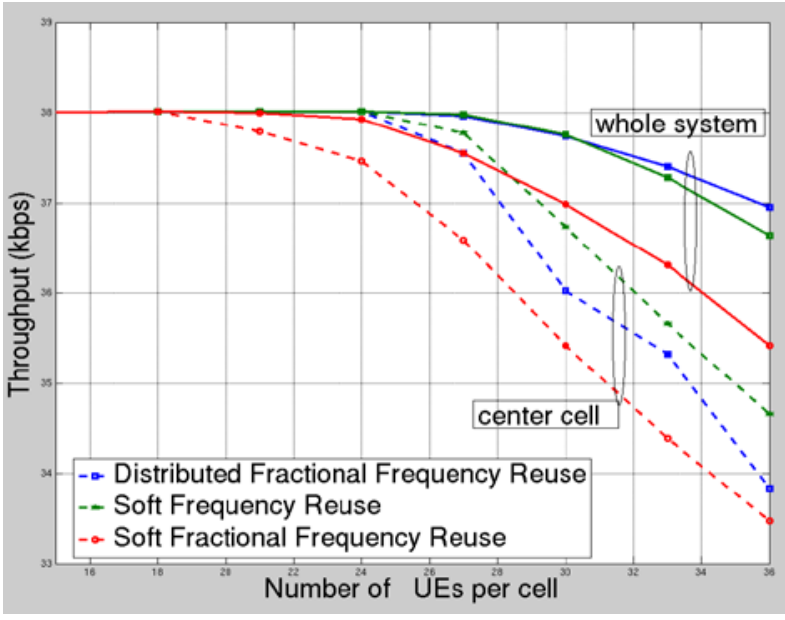

Figure 7: Average throughput per UE

\section{CONCLUSION}

This paper investigates the comparison of well-known frequency reuse algorithms in downlink 3GPP LTE system and identifies the strengths and weaknesses of these algorithms to satisfy the VoIP QoS service for the users. The differences between these algorithms are presented through the paper by both mathematical logic and simulation. Soft FFR is promised to improve the system performance without increasing the power consumption, however, splitting the whole bandwidth into 3 small parts leads to a decrease in the bandwidth flexible. Hence, it obtains the lowest system performance comparing with Distributed FFR and Soft FR. Distributed FFR archives the lowest intercell interference; however, due to the complexity of this algorithm, it only slightly outperforms Soft FR in term of whole system performance. Then it is important to conclude that reducing of complex computation of the algorithms is as important as proposing new ideas which can improve the system performance.

\section{REFERENCES}

1. R1-050507, G.P.D., Soft Frequency Reuse Scheme for UTRAN LTE. 3GPP R1-050507 Huawei, 2005.

2. Abdelbaset S. Hamza, S.S.K., Haitham S. Hamza, and Khaled Elsayed, A Survey on Inter-Cell Interference Coordination Techniques in OFDMA$\begin{array}{lcrr}\text { Based } & \text { Cellular } & \text { Networks. } & \text { IEEE } \\ \text { COMMUNICATIONS } & \text { SURVEYS } & \text { \& }\end{array}$ TUTORIALS, 2013. VOL. 15, NO. 4,.

3. Khan, F., LTE for 4G Mobile Broadband: Air Interface Technologies and Performance. 2009: Cambridge University Press, .

4. Kimura, H.S., Inter-Cell Interference Coordination (ICIC) Technology. FUJITSU Sci. Tech. J., Vol. 48, No. 12012. 
5. C802.16m-08/782, I., Fractional Frequency Reuse in Uplink. LG Electronics, 2008. Available (July 2012).

6. Soft Frequency Reuse Scheme for UTRAN LTE, C. 3rd Generation Partnership Project, France, 3GPP Project Document R1-050507, Editor.

7. Hamza, A.S., et al., A Survey on Inter-Cell Interference Coordination Techniques in OFDMABased Cellular Networks. Communications Surveys \& Tutorials, IEEE, 2013. 15(4): p. 1642-1670.

8. Kwon, Y., et al., Power Control for Soft Fractional Frequency Reuse in OFDMA System, in
Computational Science and Its Applications ICCSA 2010, D. Taniar, et al., Editors. 2010, Springer Berlin Heidelberg. p. 63-71.

9. Ns3, N.S. https://www.nsnam.org.

10.36.104, G.T., E-UTRA Base Station (BS) radio transmission and reception. 2010.

11.3GPP TS 26.236 version 10.0.0 Release 10. 2011.

12. 8, G.T.v.R., Physical layer procedures 2009.

13. V11.6.0, G.T., Policy and charging control architecture. 2012.

\begin{tabular}{|c|c|c|c|c|c|c|c|c|c|}
\hline & \multicolumn{2}{|c|}{ Strict FR } & \multicolumn{2}{|c|}{ Soft FR } & \multicolumn{3}{|c|}{ Soft FFR } & \multirow{2}{*}{\multicolumn{2}{|c|}{$\begin{array}{c}\begin{array}{c}\text { Distributed } \\
\text { FR }\end{array} \\
\text { CCU }\end{array}$}} \\
\hline & CEU & $\mathrm{CCU}$ & CEU & $\mathrm{CCU}$ & CEU & CC & CMU & & \\
\hline $\begin{array}{l}\text { Interfering } \\
\text { probability }\end{array}$ & $\frac{\Delta}{N_{C}}$ & $\frac{1}{N_{E}}$ & $\frac{1}{N_{C}}$ & $\frac{1}{N_{E}}$ & $\frac{1}{N_{C}^{\prime}}$ & $\frac{1}{N_{E}^{\prime}}$ & $\frac{1}{N_{M}}$ & $<\frac{1}{N_{C}}$ & $<\frac{1}{N_{E}}$ \\
\hline $\begin{array}{l}\text { Max number of } \\
\text { interfering BS }\end{array}$ & $\frac{M}{\Delta}$ & $M$ & $\begin{array}{l}\frac{(\Delta-}{\Delta} \\
\text { and }\end{array}$ & $\begin{array}{l}M^{(1)} \\
M^{(2)}\end{array}$ & $\begin{array}{l}\frac{(\Delta-}{\Delta} \\
\text { and }\end{array}$ & $\begin{array}{l}M^{\prime(1)} \\
M^{\prime(2)}\end{array}$ & $M$ & $\begin{array}{l}<\frac{(\Delta}{} \\
\text { and }\end{array}$ & $\begin{array}{l}\frac{1)}{M^{(1)}} \\
\frac{1}{\Delta} M^{(2)}\end{array}$ \\
\hline $\begin{array}{l}\text { Transmission } \\
\text { power of } \\
\text { interfering BS }\end{array}$ & Low & High & & & & & Medium & & $\begin{array}{l}\text { Low } \\
\text { High }\end{array}$ \\
\hline
\end{tabular}

Table 1: Comparison between some well-known frequency reuse algorithms 